\title{
Impacto da severidade da oclusopatia na qualidade de vida em escolares de 12 anos da rede estadual de ensino em uma população brasileira
}

\author{
The impact of the severity of malocclusion on the quality of life of 12-year-old students \\ in the state school system in a brazilian population
}

Mabel Cancio Hayne ${ }^{1 *}$, Ana Cláudia Lima de Oliveira Meira², Márcio Campos Oliveira

\begin{abstract}
${ }^{1}$ Mestre em Saúde Coletiva pela Universidade Estadual de Feira de Santana (UEFS); ${ }^{2}$ Mestre em Odontologia, Professora da Associação Brasileira de Odontologia - Seção Bahia (ABO-BA) ${ }^{3}$ Mestre e Doutor em Ciências Odontológicas pela Universidade Federal do Rio Grande do Norte (UFRN), Professor Titular da UEFS
\end{abstract}

\begin{abstract}
Resumo
Introdução: a má oclusão pode impactar a qualidade de vida dos indivíduos acometidos. Objetivo: avaliar a associação entre a severidade das oclusopatias e o impacto na qualidade de vida em escolares de 12 anos da rede estadual de uma população brasileira. Metodologia: através do Dental Aesthetic Index (DAI) e do questionário Oral Health Impact Profile (OHIP-14), numa amostra de 503 estudantes do município de Feira de Santana-BA, estimou-se a severidade das oclusopatias e a prevalência do impacto negativo da saúde oral na qualidade de vida, verificando associações entre essas variáveis e as condições sociodemográficas da população. Para testes estatísticos, foram utilizados: o teste t de Student ou teste de Mann-Whitney, os testes de Fisher ou do qui-quadrado e variantes, além do programa estatístico computacional GraphPad Prism, versão 6.0.3, GraphPad Software, San Diego-CA, USA. Resultados: a prevalência das oclusopatias : $(51,29 \%)[45,98-54,60]$ IC $95 \%$ e suas categorias: Leve $(48,7 \%)$, Definida $(27,8 \%)$, Severa $(15,5 \%)$ e Incapacitante (8\%) tiveram associação estatística com o impacto negativo na qualidade de vida, cuja prevalência foi de $62,23 \%$ [57,91-67,33\%] IC 95\% (313/503). Conclusão: características sociodemográficas não diferiram entre os grupos. A presença e a severidade das oclusopatias estão associadas ao impacto negativo na qualidade de vida. Quanto maior o grau de severidade dos problemas oclusais, maior o impacto negativo na qualidade de vida.
\end{abstract}

Palavras-chave: Má oclusão. Qualidade de vida. OHIP. DAI

\begin{abstract}
Introdiction: the malocclusions can impact the life quality of affected subjects. Objective: of this study was to evaluate the association between severity of malocclusion and its impact on the life quality of 12 years old students. Methods: cross-sectional study using Dental Aesthetic Index (DAI) and Oral Health Impact Profile (OHIP-14) in 12 years old students from brazilian public schools. The severity of malocclusion and prevalence of oral health's negative impact on life quality was estimated verifying the possible associations between these variables and the sociodemographic conditions. Data analysis used Student $t$ Test or the Mann-Whitney Test, to compare the differences of quantitative variables, and the Fisher test or Chi-square and its variants, for categorical data. The prevalence ratio was defined with $95 \%$ confidence intervals and $p<0.05$. Results: the prevalence of malocclusion $(50.29 \%)[45,98-54,60]$ and its categories: Mild (48.7\%), Defined (27.8\%), Severe (15.5\%), Disabling (8\%) were associated with the negative impact on life quality (62.23\%) [57,91-67,33\%] IC 95\% (313/503). Conclusion: No association between sociodemographic conditions and the impact on life quality. The presence and severity of malocclusions are associated with the negative impact on life quality. The greater the degree of severity of occlusal problems, the bigger the negative impact on the quality of life.

Keywords: Malocclusion. Life quality. OHIP. DAI.
\end{abstract}

\section{INTRODUÇÃO}

É sabido que a saúde do homem deve contemplar aspectos além das condições orgânicas. Assim, passa a ser importante considerar outras dimensões envolvidas e a repercussão das doenças na vida dos indivíduos. $\mathrm{Na}$ odontologia, ao se ignorar a subjetividade dos pacientes, existe uma tendência em tratar a cavidade oral como se fosse uma estrutura anatômica autônoma e isolada (LOCKER, 1997).

Correspondente/Corresponding: * Mabel Cancio Hayne - End: Av. Transnordestina, Novo Horizonte s/n Feira de Santana, Bahia, BR. CEP 44036-900. Tel: (75) 99144-1284 -E-mail: mahayne@hotmail.com
Entende-se como saúde bucal a condição dos tecidos orais e estruturas relacionadas, que contribuem positivamente para o bem-estar físico, mental e social e para o desfrute das possibilidades da vida, permitindo ao indivíduo falar, comer e socializar-se sem dor, desconforto ou constrangimento (CDA, 2011).

Definida como a alteração do crescimento e desenvolvimento que afeta a oclusão dos dentes, a oclusopatia ocupa o terceiro lugar na escala de prioridades entre os problemas odontológicos de saúde pública mundial, superada apenas pela cárie e pelas doenças periodontais (WHO, 1997), e pode interferir negativamente na qualidade de vida, prejudicando a 
interação social e o bem-estar psicológico dos indivíduos acometidos (OLIVEIRA; SHEIHAM, 2004). Ela pode causar impacto estético nos dentes, na face e alterar atividades funcionais (fonação, mastigação, respiração, postura) dos indivíduos, especialmente das crianças que estão em fase de socialização, podendo ser causa de baixa autoestima (FRAZÃO et al., 2002).

A qualidade de vida é algo subjetivo, ou seja, só possível de ser avaliado pelo próprio sujeito. Não há rótulos de "boa" ou "má" qualidade de vida. Logo, enfatiza-se a prioridade do subjetivismo em toda reflexão sobre esta temática (SEGRE; FERRAZ, 1997). A concepção de qualidade de vida dos indivíduos está, para alguns, dentro de conceitos que vão muito além da saúde. Por isso mesmo, a identificação de elementos que interferem diretamente na relação saúde e qualidade de vida vem despertando interesse da comunidade científica (LOCKER, 1997; MOURA; CAVALCANTI, 2007).

Para melhor avaliar a qualidade de vida, a saúde pública tem considerado a necessidade de indicadores na elaboração de suas políticas (SEGRE; FERRAZ, 1997). Os indicadores que se baseiam somente na proporção de indivíduos doentes da população tornam-se, portanto, incompletos. $\mathrm{O}$ uso de indicadores sociodentários na epidemiologia da saúde bucal tem sido amplamente defendido (LOCKER; LEÃO, 2006; SLADE, 1997). As razões para isto decorrem do fato de que as medidas clínicas, quando usadas isoladamente, não documentam todo o impacto das desordens bucais em pacientes e populações (LOCKER; LEÃO, 2006).

Nesse contexto, importa saber de que maneira a severidade da oclusopatia pode influenciar na qualidade de vida em crianças de 12 anos, causando impactos negativos, como problemas psicológicos, sociais, estéticos e funcionais. A motivação pelo tema consiste na tentativa de evitar que alguns destes agravos, tratáveis nesta idade na atenção básica, evoluam para afecções mais graves na fase adulta, necessitando assim de tratamentos cirúrgicos com custo mais elevado, além da busca por minimizar os possíveis problemas de interação social vivenciados por estas crianças.

Portanto, considera-se urgente o planejamento de meios que garantam o acesso dos casos mais severos ao tratamento corretivo especializado. Quanto à triagem para tratamento corretivo, entende-se que um índice que estratifique as oclusopatias em graus de severidade deva ser empregado em detrimento de análises subjetivas. Além disso, considerando que a presença associada de alterações funcionais é prejudicial à qualidade de vida de seus portadores, esta presença poderia ser utilizada como um critério adicional para o estabelecimento das referidas prioridades (SULIANO et al., 2007).

No tocante ao objetivo, este estudo teve o propósito de avaliar a associação entre severidade das oclusopatias e o impacto na qualidade de vida em estudantes de 12 anos das escolas estaduais de um município no estado da Bahia, Brasil.

\section{METODOLOGIA}

A amostra de 503 indivíduos foi obtida a partir de uma população de 3708 estudantes de 12 anos nas escolas estaduais do município de Feira de Santana. O cálculo amostral foi realizado com o objetivo de verificar associação entre oclusopatia e a qualidade de vida. Os parâmetros utilizados foram obtidos a partir de um estudo anterior (estudo piloto), cujos resultados apontaram para associação entre o impacto negativo na qualidade de vida e a prevalência das oclusopatias, porém não se comprovou a mesma relação com o grau de severidade. Esse fato, provavelmente, esteve relacionado ao pequeno tamanho da população estudada.

Foi utilizada a técnica de amostragem probabilística por conglomerado em único estágio e incluídas apenas as 69 escolas que possuíam estudantes na faixa etária referida acima, de ambos os gêneros. Esta idade foi selecionada por ser uma das escolhidas para monitorização global em saúde bucal nas comparações internacionais e para acompanhamento das tendências das doenças bucais, tendo sido as mesmas preconizadas no SB Brasil 2010 (BRASIL, 2010).

Foi determinado um poder de $90 \%$ e um nível de significância de $5 \%$. Obteve-se então um tamanho amostral de 185 indivíduos. Aplicando-se a correção para o efeito do desenho do estudo (DEFF) igual a 2, resultou o número de 370 indivíduos. O DEFF é um ajuste necessário, que visa a redução das imprecisões existentes devido a possíveis correlações entre os conglomerados. Ao acrescentar o percentual de $20 \%$, para se precaver de possíveis perdas de campo ou taxas de recusa, foi calculado o tamanho amostral de, no mínimo, 444 estudantes, com a média de 53,74 por escola. Finalmente, 9 escolas foram sorteadas. Foi realizado um censo em cada uma delas, todos os estudantes de 12 anos foram selecionados e convidados a participar da pesquisa, obtendo-se uma amostra final de 503 estudantes.

A coleta de dados foi realizada no próprio ambiente escolar, através do exame clínico bucal registrado conforme o Índice de Estética Dental - Dental Aesthetic Index (DAI), da aplicação do questionário Perfil do Impacto da Saúde Oral (OHIP-14) e do Formulário para os pais (MEIRA, 2010), com questões sociodemográficas, incluindo variáveis como gênero, raça/cor da pele, renda familiar, escolaridade dos pais, atendimento odontológico no último ano, tipo de serviço do atendimento, uso de aparelho ortodôntico, tipo de apareIho ortodôntico, local de atendimento do tratamento ortodôntico (serviço público ou privado).

Foram calculados os índices Kappa para cada condição avaliada no DAI durante o levantamento, com um grupo representando $10 \%$ da amostra, em adição à calibração inicial do examinador em estudo anterior (estudo piloto). Os resultados demonstraram excelente reprodutibilidade de diagnóstico, conforme tabela 1. 
Tabela 1 - Índice Kappa intra-examinador antes e durante a coleta para os critérios componentes do índice DAI.

\begin{tabular}{lcc}
\hline \multicolumn{1}{c}{ Índice Kappa } & Calibração inicial & Reexame \\
\hline Dentição Superior & 1,00 & 1,00 \\
Dentição Inferior & 1,00 & 1,00 \\
Apinhamento & 0,90 & 0,94 \\
Espaçamento & 0,89 & 1,00 \\
Diastema & 1,00 & 1,00 \\
Desalinhamento maxilar & 0,89 & 0,95 \\
Desalinhamento mandibular & 0,84 & 0,97 \\
Sobressaliência maxilar & 0,95 & 0,97 \\
Sobressaliência mandibular & 1,00 & 0,91 \\
Relação molar & 1,00 & 1,00 \\
Mordida aberta & 1,00 & 1,00 \\
\hline
\end{tabular}

Fonte: Dados da pesquisa

O questionário OHIP-14 avalia, através de 14 perguntas, o impacto na qualidade de vida sob o aspecto de sete dimensões: limitação funcional, dor física, desconforto psicológico, incapacidade física, incapacidade psicológica, incapacidade social e deficiência. As respostas são marcadas considerando-se uma escala de 05 pontos, na qual código $0=$ "nunca"; código $1=$ "poucas vezes"; código 2 = "às vezes"; código $3=$ "quase sempre"; código 4 = "sempre". Para cada pergunta é atribuído um peso. As respostas produziram a soma dos valores absolutos, aqui denominada OHIP absoluto, e também a soma dos valores multiplicados pelos pesos de cada questão, o OHIP escores. A partir deste ponto, foram estabelecidas as medianas da amostra total. Para a análise estatística, um dado dicotômico foi obtido através da presença de uma ou mais respostas com os códigos 3 e 4, indicando as pessoas que tinham experimentado pelo menos algum impacto (SLADE, 1997).

Os critérios clínicos para determinar a necessidade normativa de tratamento ortodôntico foram determinados pelo DAl, índice recomendado pela Organização Mundial da Saúde (WHO, 1997). Este inclui a avaliação das seguintes condições: ausência de dentes superiores e inferiores, presença de diastemas ou de apinhamentos nos segmentos anteriores superiores e inferiores, overjet maxilar e mandibular anterior, mordida aberta anterior e relação molar ântero-posterior. Os indivíduos na fase de dentição mista foram excluídos do estudo, pois poderiam ocasionar equívoco para a determinação do DAI (WHO, 1997; MEIRA, 2010).
Após a avaliação e adequação dos cálculos dos valores obtidos, o DAl fornece quatro possibilidades de desfecho: ausência de anormalidade ou oclusopatias leves, com nenhuma ou pouca necessidade de tratamento ortodôntico (DAI $\leq 25$ ); oclusopatia definida, cujo tratamento é eletivo (DAI = 26-30); oclusopatia severa, cujo tratamento é altamente desejável (DAI = 31 a 35); e oclusopatia muito severa ou incapacitante, cujo tratamento ortodôntico é fundamental (DAI $\geq 36$ ). Essa variável foi dicotomizada em: sem necessidade de tratamento (DAI $\leq 25$ ) e com necessidade de tratamento (DAI > 25) (GARBIN et al., 2010).

As variáveis quantitativas foram descritas por suas medidas de tendência central (médias ou medianas) e pelas respectivas medidas de dispersão (desvio-padrão ou variação interquartil), enquanto as nominais ou qualitativas foram descritas por seus valores absolutos, percentagens ou proporções. Para comparação das diferenças das variáveis quantitativas, foi utilizado o teste $t$ de Student ou o teste de Mann-Whitney. O Teste de D'Agostino-Pearson foi empregado para avaliação do padrão de distribuição das variáveis. Na comparação dos dados categóricos, foram utilizados os testes de Fisher ou do qui-quadrado e suas variantes.

A distribuição das covariáveis não apresentou diferenças na análise univariada, nem no modelo de análise multivariada, para identificação de possíveis preditores independentes. A associação entre os parâmetros estudados foi expressa pela razão de prevalência. Intervalos de confiança de $95 \%$ foram empregados como medidas de precisão dos resultados. Valores de $p$ menores que $0,05(p<0,05)$ foram considerados significativos. Nas análises, foi utilizado o programa estatístico computacional GraphPad Prism, versão 6.0.3, GraphPad Software, San Diego-CA, USA.

\section{RESULTADOS}

O grupo examinado é composto por 503 estudantes de 12 anos, em sua maioria negros e com discreta predominância de meninos. Do ponto de vista estatístico, neste trabalho foram agregadas as pessoas pretas e pardas num único grupo dos negros. A maioria dos sujeitos não possui plano de atendimento odontológico, nem tampouco receberam atendimento no último ano. Quando atendida, a discreta maioria recebeu o atendimento em caráter privado. As características sociodemográficas da população estudada estão descritas na tabela 2 . 
Tabela 2 - Características sociodemográficas de estudantes de 12 anos da rede estadual de ensino. Feira de Santana-BA, 2015.

\begin{tabular}{lcc}
\hline \multicolumn{1}{c}{ Variável } & $\mathbf{n}$ & $\%$ \\
\hline Gênero & 263 & 52,3 \\
$\quad$ Masculino & 240 & 47,7 \\
$\quad$ Feminino & & \\
Raça/ Cor da Pele & 105 & 20,9 \\
$\quad$ Brancos & 398 & 79,1 \\
$\quad$ Negros (Pretos e Pardos) & & \\
Plano Odontológico & 434 & 86,3 \\
$\quad$ Não possui & 69 & 13,7 \\
$\quad$ Possui & & \\
Atendimento no último ano & 357 & 71 \\
$\quad$ Não & 146 & 29 \\
$\quad$ Sim & & \\
Local do atendimento & 63 & 43,2 \\
$\quad$ Serviço público & 83 & 56,8 \\
$\quad$ Serviço privado & & \\
MEDIANA [IIQ] & & \\
Escolaridade dos pais* & 11 [8-12] \\
Renda** & 804 [788-1500] & \\
\hline
\end{tabular}

*Valor em anos de estudo.

** Valor em Reais.

Fonte: Dados da pesquisa

A prevalência das oclusopatias foi de 51,29\% [45,98-54,60] IC 95\%. Dos 258 sujeitos com disfunção oclusiva, somente 28 usavam aparelho ortodôntico. Em sua maioria, 96,4\% (27/28), o tratamento era feito em serviços privados. A severidade das oclusopatias está descrita na tabela 3.

Tabela 3-Severidade das oclusopatias em escolares de 12 anos da rede estadual de ensino. Feira de Santana-BA, 2015.

\begin{tabular}{llrr}
\hline Variável & \multicolumn{1}{c}{ Categorias } & $\mathbf{n}$ & $\%$ \\
\hline \multirow{4}{*}{ DAI } & Ausente/ Leve & 245 & 48,7 \\
& Definida & 140 & 27,8 \\
& Severa & 78 & 15,5 \\
& Incapacitante & 40 & 8,0 \\
\hline
\end{tabular}

Fonte: Dados da pesquisa

A prevalência do impacto negativo da saúde oral na qualidade de vida foi de 62,23\% [57,91-67,33\%] IC 95\% (313/503). Na amostra, o valor do OHIP absoluto (soma dos resultados ordinais) foi de 10 [6-14] e o do OHIP escores (soma dos resultados multiplicados ao seu escore) foi de 5 [3-7.2]. As características sociodemográficas não diferiram nos dois grupos e estão mais bem detalhadas na tabela 4.
Tabela 4 - Características sociodemográficas dos estudantes de 12 anos com e sem impacto negativo na qualidade de vida. Feira de Santana-BA, 2015.

\begin{tabular}{|c|c|c|c|}
\hline Variável & $\begin{array}{l}\text { Impacto } \\
\text { Negativo }\end{array}$ & Sem Impacto & $p$ \\
\hline $\mathrm{N}(\%)$ & $313(62,2 \%)$ & $190(37,8 \%)$ & \\
\hline \multicolumn{4}{|l|}{ Gênero } \\
\hline Feminino & $149(47,6 \%)$ & $91(47,9 \%)$ & 1,000 \\
\hline Masculino & $164(52,4 \%)$ & $99(52,1 \%)$ & \\
\hline \multicolumn{4}{|l|}{ Raça/Cor da Pele } \\
\hline Negros & $252(80,5 \%)$ & $146(76,8 \%)$ & 0,365 \\
\hline Brancos & $61(19,5 \%)$ & $44(23,2 \%)$ & \\
\hline \multicolumn{4}{|l|}{ Plano Odontológico } \\
\hline Sim & $50(16 \%)$ & $19(10 \%)$ & 0,062 \\
\hline Não & 263 (84\%) & $171(90 \%)$ & \\
\hline \multicolumn{4}{|c|}{ Atendimento último ano } \\
\hline Sim & $100(32 \%)$ & $46(24,2 \%)$ & 0,069 \\
\hline Não & $213(68 \%)$ & $144(75,8 \%)$ & \\
\hline \multicolumn{4}{|l|}{ MEDIANA [IIQ] } \\
\hline Renda Familiar* & 804 [788-1500] & 800 [788-1280] & 0,075 \\
\hline Escolaridade** & 11 [8-12] & 11 [9-12] & 0,741 \\
\hline
\end{tabular}

A associação entre a presença de oclusopatias, a severidade dos casos, o tratamento ortodôntico e o impacto na qualidade de vida está demonstrada na tabela 5 .

Tabela 5 - Associação entre a presença e a severidade das oclusopatias, o seu tratamento e o impacto na qualidade de vida em estudantes de 12 anos. Feira de Santana-BA, 2015.

\begin{tabular}{|c|c|c|c|c|}
\hline & $\begin{array}{l}\text { Impacto } \\
\text { Negativo }\end{array}$ & $\begin{array}{c}\text { Sem } \\
\text { Impacto }\end{array}$ & RP & $\mathbf{p}$ \\
\hline \multicolumn{5}{|l|}{$\begin{array}{l}\text { Presença de } \\
\text { oclusopatias }\end{array}$} \\
\hline Sim & $174(67,4 \%)$ & $84(32,6 \%)$ & $1,19[1,03-1,35]$ & 0,0167 \\
\hline Não & $139(56,7 \%)$ & $106(43,3 \%)$ & & \\
\hline \multicolumn{5}{|l|}{$\begin{array}{l}\text { Severidade } \\
\text { das } \\
\text { Oclusopatia }\end{array}$} \\
\hline $\begin{array}{l}\text { Ausente/ } \\
\text { Leve }\end{array}$ & $139(56,7 \%)$ & $106(43,3 \%)$ & 1,00 & 0,0375 \\
\hline Definida & $90(64,3 \%)$ & $50(35,7 \%)$ & $1,13[0,96-1,34]$ & \\
\hline Severa & $53(67,9 \%)$ & $25(32,1 \%)$ & $1,19[0,99-1,44]$ & \\
\hline $\begin{array}{l}\text { Incapaci- } \\
\text { tante }\end{array}$ & $31(77,5 \%)$ & $9(22,5 \%)$ & $1,37[1,12-1,67]$ & \\
\hline \multicolumn{5}{|l|}{$\begin{array}{l}\text { Uso de } \\
\text { aparelho } \\
\text { ortodôntico }\end{array}$} \\
\hline $\operatorname{Sim} *$ & $18(64,3 \%)$ & $10(35,7 \%)$ & $1.03[0,78-1,38]$ & 1,000 \\
\hline Não** & $295(62,1 \%)$ & $180(37,9 \%)$ & & \\
\hline
\end{tabular}

* Sim: são consideradas as oclusopatias definida, severa e incapacitante com necessidade de tratamento.

**Não: são consideradas ausência de oclusopatia ou oclusopatias leves com nenhuma ou pouca necessidade de tratamento.

Fonte: Dados da pesquisa 


\section{DISCUSSÃO}

O maior interesse pela relação entre saúde e vida com qualidade pode ser percebido pela quantidade e diversidade de estudos que vêm sendo publicados nas últimas décadas (OLIVEIRA; SHEIHAM, 2004; MOURA; CAVALCANTI, 2007; FEU et al., 2008; ALMEIDA et al., 2014; GATTO et al., 2019; PETER et al., 2019). As oclusopatias apresentam uma alta prevalência na população (FRAZÃO et al., 2002), podendo influenciar negativamente a qualidade de vida dos indivíduos acometidos (FEU et al., 2008), apresentando possibilidades de alterações funcionais (SULIANO et al., 2007) e insatisfação com a aparência dentofacial dos indivíduos (ALMEIDA et al., 2014).

No presente estudo, características sociodemográficas observadas na amostra, como gênero, raça/cor da pele (LOPES; CANGUSSU, 2005), escolaridade dos pais e renda familiar (LOPES; CANGUSSU, 2005; MEIRA et al., 2011), corroboraram com outros estudos. A ausência de significância estatística entre essas variáveis e o impacto negativo na qualidade de vida (FEU et al., 2008) podem evidenciar a multifatorialidade desta temática. Observou-se que as variáveis plano odontológico, atendimento odontológico no último ano e renda familiar aproximaram-se da significância estatística, mesmo sendo a amostra homogênea (apenas estudantes da rede pública). Isto aponta para a importância das variáveis sociodemográficas na qualidade de vida dos indivíduos acometidos por oclusopatias. Por isso mesmo, as características socioeconômicas dos pacientes que buscam tratamento ortodôntico não devem ser negligenciadas (PETER et al., 2019).

Poucos estudantes usam ou usaram algum tipo de aparelho ortodôntico. Desses, a maioria utilizou fixo/ corretivo e apenas um indivíduo dirigiu-se à rede pública para colocação da aparatologia. Este fato pode estar relacionado à deficiência de programas diretamente direcionados às oclusopatias na rede básica de saúde do município. Com base neste estudo, fica evidente a necessidade de organização dos programas e serviços de saúde para a implementação de medidas que visem prevenir, interceptar e tratar as oclusopatias, em especial as severas e as incapacitantes (MEIRA et al., 2011).

Os resultados apontam para uma associação positiva e estatisticamente significativa $(p=0,0167)$ entre as oclusopatias e o impacto negativo na qualidade de vida em estudantes de 12 anos da rede estadual de ensino no município de Feira de Santana. Esse tipo de resultado também aconteceu em outro estudo com adolescentes (FEU et al., 2008).

Em contrapartida, dos 245 indivíduos com oclusopatia ausente ou leve (com nenhuma ou pouca necessidade de tratamento), $56,7 \%$ deles relataram impacto negativo na qualidade de vida. Podemos inferir, que, provavelmente, como o OHIP mensura a qualidade de vida em relação à saúde oral dos indivíduos e não apenas as oclusopatias, o impacto negativo na qualidade de vida desses indivíduos pode estar relacionado a outras dimensões da saúde bucal.

A alta prevalência $(51,29 \%)$ encontrada corrobora com outras pesquisas de indivíduos na mesma faixa etária: 71,31\% em Frazão et al. (2002); 45,8\% no estudo de Lopes e Cangussu (2005); 78,4\% em Moura e Cavalcanti (2007); $82,1 \%$ em Suliano et al. (2007). Também no município de Feira de Santana, outro estudo relatou prevalência elevada (53\%) das oclusopatias aos 12 anos (MEIRA et al., 2011). No Brasil, essa porcentagem foi de $38 \%$ em 2010 (BRASIL, 2010).

Ao estratificar as oclusopatias em definida $(27,8 \%)$, severa (15,5\%) e incapacitante ( $8 \%)$, o estudo apresentou valores epidemiológicos altos, como ocorreu em outros estudos (MEIRA et al., 2011; MOURA; CAVALCANTI, 2007; SULIANO et al, 2007;), com algumas variações nas porcentagens. Grande parte da amostra $(62,23 \%)$ teve impacto negativo na qualidade de vida, corroborando com outro estudo feito com adolescentes portadores de oclusopatias, cuja prevalência foi de $43 \%$, segundo OHIP14 (OLIVEIRA; SHEIHAM, 2004). Ao associar as categorias das oclusopatias (definida, severa e incapacitante) aos resultados do OHIP-14, houve significância estatística, ou seja, os achados sugerem que quanto maior a severidade das oclusopatias, maior o impacto negativo na qualidade de vida, corroborando com Paula et al. (2009).

$\mathrm{O}$ termo qualidade de vida abrange muitos significados, que refletem conhecimentos, experiências e valores individuais e subjetivos. Indivíduos que procuram o tratamento ortodôntico tendem a apresentar impacto negativo em sua qualidade de vida, oclusopatias mais graves (FEU et al., 2008) e pior impacto estético (FEU et al., 2008; ALMEIDA et al., 2014). A varável uso de aparelho ortodôntico não foi adotada como critério de exclusão, pois para a pesquisa importa saber se indivíduos com necessidade de tratamento e que tiveram acesso ao uso do aparelho ortodôntico são capazes de apresentar maior impacto da sua condição oclusal na qualidade de vida.

Porém, no presente estudo não houve associação entre o uso do aparelho ortodôntico e os resultados do OHIP-14. Este fato pode estar relacionado ao pequeno número de individuos na amostra que usam ou usaram a aparatologia. Tal achado diverge da maioria dos estudos, que apontam para uma melhor qualidade de vida dos sujeitos submetidos ao tratamento ortodôntico completo, comparados àqueles que ainda se encontram em tratamento ou nunca o realizaram (FEU et al., 2008; GATTO et al, 2019; OLIVEIRA; SHEIHAM, 2004).

O diagnóstico precoce e o tratamento das oclusopatias são demandas existentes na população. A maioria desses agravos, tratáveis durante a infância, podem evoluir para afecções mais graves na fase adulta, necessitando de tratamentos de média e alta complexidade. Programas ortodônticos preventivos e interceptativos podem ser utilizados, no serviço público, para ações relacionadas ao tratamento precoce das oclusopatias, sendo possível também a implantação de procedimentos 
em Ortodontia Corretiva. O desenvolvimento de protocolos de tratamento e a capacitação profissional de toda a equipe, sobretudo dos cirurgiões-dentistas, podem ser colocados em prática, visando à prevenção da progressão das desarmonias dentárias, esqueléticas e funcionais (HEBLING et al., 2007).

Sendo assim, é de fundamental importância o desenvolvimento de políticas públicas que visem à inserção do tratamento ortodôntico. É inegável a importância desse tipo de tratamento entre os procedimentos dos programas de saúde na Atenção Básica, bem como a sua implementação nos centros odontológicos especializados.

\section{CONCLUSÃO}

A partir dos achados deste estudo, evidenciou-se que a presença e a severidade das oclusopatias estão associadas ao impacto negativo na qualidade de vida em estudantes de 12 anos da rede estadual de ensino da população estudada. Quanto maior o grau de severidade dos problemas oclusais, maior o impacto negativo na qualidade de vida. Não obstante, mesmo com ausência/ oclusopatia leve, indivíduos desta amostra também apresentaram impacto negativo na qualidade de vida. Já as condições sociodemográficas não apresentaram associações estatísticas com as variáveis principais do estudo.

\section{REFERÊNCIAS}

ALMEIDA, A. B. et al. Dissatisfaction with dentofacial appearance and the normative need for orthodontic treatment: determinant factors. Dental Press J. Orthod., Maringá, v.19, n.3, p. 120-126, 2014.

BRASIL, Ministério da Saúde. Secretaria de Vigilância em Saúde. Secretaria de Assistência á Saúde. Departamento de Atenção Básica. Coordenação Nacional de Saúde Bucal. Pesquisa Nacional de Saúde Bucal 2010. Brasília, 2010.

CDA Canadian Dental Association. communiqué Junho 2011. Disponível em: http://www.cdaadc.ca/en/oral_health/index.asp. Acesso em: 29 de junho 2011.

FEU, D. et al. Más-oclusões e seu impacto na qualidade de vida de adolescentes que buscam tratamento ortodôntico. Ortodontia SPO, São Paulo, v. 41, n. 4, p.355-65, 2008.

FRAZÃO, P. et al. Prevalência de oclusopatia na dentição decídua e permanente de crianças na cidade de São Paulo, Brasil, 1996. Cad. Saúde Pública, Rio de Janeiro, v. 18, n. 5, p. 1197-1205, 2002.

GARBIN, A. J. I. et al. Prevalência de oclusopatias e comparação entre a Classificação de Angle e o Índice de Estética Dentária em escolares do interior do estado de São Paulo - Brasil. Dental Press J. Orthod., Maringá, v.15, n. 4, p 94-102, 2010.
GATTO, R. C. J. et al. Qualidade de vida relacionada à saúde bucal, necessidade de tratamento ortodôntico e bullying entre adolescentes brasileiros. Dental Press J. Orthod., Maringá, v.24, n.2, p. 73-80, 2019.

HEBLING, S. R. F. et al. Considerações para elaboração de protocolo de assistência ortodôntica em saúde coletiva. Ciênc. Saúde Colet., Rio de Janeiro, v.12, n. 4, p.1067-1078, 2007.

LOCKER, D. Concepts of Oral Health, Disease and the Quality of Life. In: SLADE G. D. Measuring Oral Health and Quality of Life, Chapel Hill: University of North Carolina, Dental Ecology, 1997. cap 2. p. 11-23.

LOCKER, D.; LEÃO, A. T. Impacto nas condições de Saúde bucal na qualidade de vida. In: ANTUNES, J. L. F.; PERES, M. A. Fundamentos de Odontologia, Epidemiologia da Saúde Bucal. Rio de Janeiro, Ed. Guanabara Koogan: 2006. p 260-267.

LOPES, L. S.; CANGUSSU, M. C. T. Prevalência e severidade das alterações oclusais em escolares de 12 a 15 anos de Salvador- BA, 2004. R. Ci. méd. biol., Salvador, v. 4, n. 2, p. 105-112, 2005.

MEIRA, A. C. L. de O. Oclusopatias em crianças e adolescentes escolares no município de Feira de Santana, Bahia. Feira de Santana, 2010 [Dissertação de Mestrado - Universidade Estadual de Feira de Santana].

MEIRA, A. C. L. O.; OLIVEIRA, M. C.; ALVES, T. D. B. Severidade das oclusopatias e fatores associados em escolares de 12 anos no município de Feira de Santana, Bahia, 2009. Rev. Baiana de Saúde Pública, Salvador, v. 35, supl.1, p.196-210, 2011.

MOURA C.; CAVALCANTI, A. L. Maloclusões, cárie dentária e percepções de estética e função mastigatória: um estudo de associação. Rev. Odonto Ciênc., Porto Alegre, v. 22, n. 57, p. 256-262, 2007.

OLIVEIRA C. M.; SHEIHAM A. Orthodontic treatment and its impact on oral health related quality of life in Brazilian adolescents. J. Orthod., London, v. 31, p. 20-7, 2004.

PAULA, D. F. D. J. et al. Psychosocial Impact of Dental Esthetics on Quality of Life in Adolescents, Association with Malocclusion, Self-Image, and Oral Health-Related Issues. Angle Orthod., Aplleton, v. 79, n. 6, 2009.

PETER, E. et al. Questionário “Impacto da Má Oclusão na Qualidade de Vida" (MRQoLQ): desenvolvimento e validação de uma nova ferramenta psicométrica para adolescentes. Dental Press J. Orthod.,. Maringá, v. 24, n. 6, 2019.

SEGRE, M.; FERRAZ, F.C. O conceito de saúde. Rev. Saúde Pública, v. 31, n. 5, p. 538-542, 1997.

SLADE, G. D. Derivation and validation of a short-form oral health impact profile. Community Dentistry and Oral Epidemiology, Copenhagen, v.25, n.4, p.284-290, 1997.

SULIANO, A. A. et al. Prevalência de Maloclusão e sua associação com alterações do sistema estomatognático entre escolares. Cad. Saúde Pública, Rio de Janeiro, v. 23, n. 8, p. 1913-1923, 2007.

WORLD HEALTH ORGANIZATION. Oral health surveys, basic metods. 4th edition. Geneva: WHO, 1997.

Submetido em: 04/05/2020

Aceito em: 27/10/2020 